\title{
¿Se sienten valorados los profesores en Chile?
}

\section{Carla Hernández-Silva \\ orcid.org/0000-0002-4479-6632 \\ Universidad de Santiago, Chile \\ carla.hernandez.s@usach.cl}

\section{Alexis González-Donoso}

orcid.org/oooo-0oo1-6991-1580

University of British Columbia, Canadá

alexis.gonzalezdo@usach.cl

\section{Amaya Pavez-Lizarraga}

orcid.org/00oo-0002-4671-2092

Universidad de Santiago, Chile

amaya.pavez@usach.cl

\section{Silvia Tecpan-Flores}

orcid.org/0ooo-0001-9343-6863

Universidad de Santiago, Chile

silvia.tecpan@usach.cl

\section{Resumen}

En este artículo se presentan los resultados de un estudio exploratorio, cuyo objetivo fue conocer la percepción que tiene el profesorado chileno sobre su rol y valoración social. Se diseñó una encuesta con 21 items tipo Likert, distribuidos en dos escalas de análisis; una interna, relacionada con el rol del profesional y su aporte a la comunidad educativa, y otra externa, referida al impacto de su trabajo profesional docente en la sociedad. La consistencia interna del instrumento fue evaluada con alfa de Cronbach. Participaron 224 profesores de las 15 regiones de Chile en 2015, de distintas áreas de conocimiento y de contextos educativos diversos. Los resultados indican que existe una percepción positiva en la dimensión interna vinculada con la satisfacción personal del ser docente y la formación disciplinar del profesorado. Sin embargo, en la dimensión externa se evidenció que la percepción de los docentes frente al reconocimiento y confianza del Estado sobre su labor es negativa, aspecto que es contrastado con la alta valoración que perciben de sus estudiantes y comunidad cercana.

\section{Palabras clave}

Estado y educación evaluación del docente; identidad; apreciación de la actuación; sistema educativo; Chile (Fuente: Tesauro de la Unesco).

Recepción: 2016-12-22 | Envío a pares: 2017-05-22 | Aceptación por pares: 2017-08-06 | Aprobación: 2017-08-10 


\title{
Do Teachers in Chile Feel Appreciated?
}

\begin{abstract}
The article presents the results of an exploratory study designed to understand how Chilean teachers perceive their role and value to society. A survey was designed with 21 Likert-type items, distributed into two scales for analysis; an internal one, related to the teacher's role and his or her contribution to the educational community, and an external one, related to the impact their professional work as teachers has on society. The internal consistency of the instrument was evaluated with Cronbach's alpha. In all, 224 teachers from 15 regions in Chile took part in the study during 2015. They represented different areas of knowledge and different educational contexts.The results indicate there is a positive perception in the internal dimension linked to the teacher's personal satisfaction and the disciplinary training the teaching staff receives. However, in the external dimension, it was evident that teachers' perception of the State's recognition and trust in their work is negative. This aspect contrasts with the high degree of appreciation they perceive from their students and the surrounding community.
\end{abstract}

\section{Keywords}

State and education; teacher evaluation; identity, appreciation of performance; educational system; Chile (Source: Unesco Thesaurus). 


\section{Os professores no Chile se sentem valorizados?}

\section{Resumo}

Apresentam-se os resultados de um estudo exploratório, cujo objetivo foi conhecer a percepção que o professorado chileno tem sobre seu papel e valorização social. Elaborou-se um questionário com 21 itens, tipo Likert, distribuídos em duas escalas de análise: uma interna, relacionada com o papel do profissional e sua contribuição para a comunidade educativa; outra externa, referente ao impacto de seu trabalho docente na sociedade. A consistência interna do instrumento foi avaliada com alfa de Cronbach. O estudo foi realizado em 2015 e teve a participação de 224 professores das 15 regiões do Chile, de diferentes áreas do conhecimento e contextos educativos. Os resultados indicam que existe uma percepção positiva na dimensão interna vinculada com a satisfação pessoal de ser docente e com a formação disciplinar do professorado. Contudo, na dimensão externa, evidenciou-se que a percepção dos docentes diante do reconhecimento e confiança do Estado sobre seu trabalho é negativa, aspecto contrastado com a alta valorização que percebem de seus estudantes e da comunidade.

\section{Palavras-chave}

Apreciação da atuação; avaliação do docente; Chile; Estado e educação; identidade; sistema educativo (Fonte: Tesauro da Unesco). 


\section{Introducción}

En los últimos años el sistema educativo en Chile ha experimentado cambios producto de diversas reformas que buscan modificar la trayectoria laboral docente, el sistema de acceso a los estudios de nivel superior y la elaboración de estándares para la formación del profesorado, entre otros aspectos.' Este escenario ha generado descontento en estudiantes y profesores, quienes se han manifestado respecto a las condiciones laborales actuales, el acceso a la educación y los sistemas de evaluación impuestos a los docentes para evidenciar sus competencias profesionales (Córdoba, 2014; Flores y Retamal, 2012), entre otros criterios, al igual que en reformas educativas anteriores donde no se han sentido partícipes o escuchados (Campodónico, 2007). Así mismo, los diversos mecanismos utilizados para medir la calidad de los docentes (Pedraja, Araneda, Rodríguez y Rodríguez, 2012) y el propio conocimiento de los estudiantes (Ortiz, 2012) parecen traslucir signos de desconfianza social respecto al propio sistema educativo, y cuyos resultados pueden repercutir en el desempeño de los distintos actores involucrados.

En este escenario, resulta cuestionable que en la toma de decisiones sobre educación en Chile los docentes sean los últimos en ser consultados, sobre todo si consideramos que el rol del profesorado es clave para el éxito de cualquier reforma educativa, dada la formación pedagógica recibida (Abell, 2000). Además, son ellos quienes tienen contacto directo con los estudiantes y concretan las políticas públicas desde el nivel macrocurricular al nivel microcurricular en el aula (Robalino, 2005). La consideración que se debe tener con el profesor en este tipo de procesos también se justifica por la manera como puede verse afectada la propia percepción de los docentes sobre sus capacidades, para ejecutarlas y llevarlas a cabo, siendo este un "foco de conflicto

$1 \quad$ Para consultar documentos que regulan las reformas vigentes en los temas señalados, se sugiere visitar el sitio Web de la Comisión Nacional de Educación (www.cned.cl). en torno a la identidad docente" (Ávalos, Cavada, Pardo y Sotomayor, 2010).

Con estos antecedentes, como objetivo para el estudio se planteó explorar la percepción del profesorado chileno en ejercicio respecto a su rol docente, su desempeño y su valoración social. Se presentan a continuación algunas consideraciones teóricas sobre el rol y valoración social del profesorado, para luego describir las características metodológicas del estudio e instrumento utilizado, finalizando con el análisis de los resultados obtenidos y las implicaciones para el sistema educativo y políticas públicas.

\section{Valoración social del profesorado en Chile}

Hasta mediados de los años cincuenta, Chile era noticia por la calidad de las instituciones de formación de profesores, siendo un ejemplo de ello la Facultad de Filosofía y Educación de la Universidad de Chile, la cual se convirtió en un modelo de formación de profesores de educación secundaria de calidad en la región (Ávalos, 2002). Sin embargo, a partir de la década del setenta, la labor docente se deterioró gradualmente, al implantarse una reforma del sistema educativo con políticas que desprestigiaron la carrera de pedagogía (Donoso, 2008a). Lo anterior masificó la oferta formativa y les quitó la exclusividad a las universidades, con lo cual disminuyó la exigencia de selección para el ingreso a este tipo de carreras y la calidad de los estudiantes que ingresaban al sistema universitario (Ávalos, 2001). Paulatinamente la valoración social de la profesión también se vio afectada, por una parte, por la creencia de que quien estudiaba pedagogía pertenecía al grupo de rendimiento académico mediobajo, con bajo puntaje en la prueba estandarizada de acceso a la universidad, y por otra, por la escasa retribución económica que reciben los docentes en su labor (Rubilar, 2015).

No obstante, un estudio realizado en Chile en los años noventa (Arancibia, 1994) reveló que la percepción de la opinión pública sobre el profesorado 
era positiva, ya que la mayoría de los entrevistados consideraba que este tenía una buena formación y valoraba su trabajo. Sin embargo, los expertos consultados opinaron lo contrario: que los profesores no solo tenían mala formación, sino que además no se preocupaban por actualizarse. Si bien el estudio no indagó por las razones de tal diferencia y sí puso de manifiesto los contrastes en la percepción sobre la profesión docente en la opinión del público general, de una parte, y los expertos en educación, de la otra, no la contrastó con la opinión del propio profesorado. En esta línea, un estudio posterior (Ávalos y Sevilla, 2010) que analizó la construcción de la identidad profesional de los docentes desde su perspectiva, reveló que el grado de satisfacción del profesorado con sus capacidades pedagógicas dependía de los años de servicio y experiencia laboral, siendo los profesores que llevan más años ejerciendo la profesión los que se sentían más satisfechos con sus capacidades. No obstante, respecto al estatus social de la profesión, la percepción era mayormente negativa en todos los grupos encuestados, al no escoger la "valoración social" como un aspecto que pudiera definir la profesión docente.

Si bien, tal y como plantea Vaillant (2010), "es difícil medir el reconocimiento de una profesión por parte de la sociedad y más aún su prestigio social", para fines de esta investigación se coincide con la autora en que la "valoración social" es un estatus del que goza el profesorado, si se considera que su labor es apreciada por la sociedad e importante para ella. Además, la importancia de indagar en el tema radica en que el éxito del sistema educativo de un país se relaciona directamente con la alta valoración social del profesorado (Cabezas y Claro, 2011).

Al parecer, los constantes cambios a los que el sistema educativo chileno se ha enfrentado históricamente han provocado que el estatus del profesorado se vea alterado y cuestionado, con la consiguiente crisis en la labor docente y en su valoración social. Esto último debido principalmente a que el docente pareciera no ser capaz de adaptarse a los cambios e ir acorde a las actuales necesidades que el sistema educativo impone (Arévalo, Cornejo, Reyes y Sánchez, 2010). Desde esta perspectiva, la presente investigación estudia cómo se percibe el docente actualmente en Chile, entendiendo el concepto de percepción como "el proceso cognitivo de la conciencia que consiste en el reconocimiento, interpretación y significación para la elaboración de juicios en torno a las sensaciones obtenidas del ambiente físico y social" (Melgarejo, 1994).

Basándose en el componente subjetivo y social de la percepción, se diferencian dos componentes en la percepción del profesorado: uno interno, referido al impacto que tiene en la formación de sus estudiantes la propia formación profesional recibida y su participación como agente clave del sistema educativo; y otro externo, que considera el reconocimiento social recibido por parte del Estado, la sociedad y la comunidad educativa cercana. Ambos niveles se proponen como perspectivas complementarias y necesarias para la construcción de la identidad profesional (Novoa, 2009).

\section{Figura 1. Aspectos que conforman la identidad profesional y la percepción del profesorado}

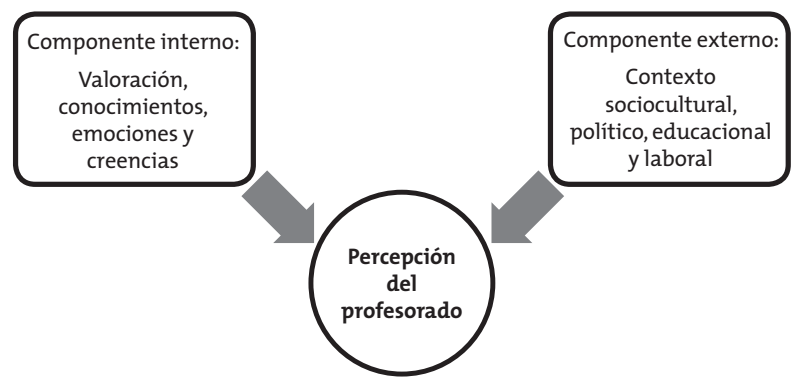

Fuente: adaptado de Ávalos et al. (2010).

\section{Metodología}

Se realizó un estudio exploratorio, no experimental, transeccional con una muestra por conveniencia empleando el método de encuesta a través de un cuestionario autoadministrado en formato electrónico (Hernández, Fernández y Baptista, 2006). El tipo de estudio favoreció la pesquisa de un 
aspecto relevante del sistema educacional chileno, poco estudiado en el país, como es la significación y opinión que tienen los docentes sobre su valoración social. Además, la técnica de recolección de la información permitió acceder a profesores de todas las regiones, edad, sexo y tipo de institución, según la administración del establecimiento.

\section{Instrumento}

Para la recolección de datos se construyó un cuestionario que fue validado por constructo y contenido a través del juicio de expertos (Valenzuela y Flores, 2011). Participaron tres expertos en educación externos al estudio y tres profesores de colegio en ejercicio. Cada uno de los seis revisores entregó por escrito sus comentarios y sugerencias respecto a cada ítem, con base en una pauta de revisión. A partir de los resultados obtenidos se hicieron los ajustes necesarios para mejorar la comprensión, redacción y objetivo de las preguntas. Al término de este procedimiento se analizó la confiabilidad con alfa de Cronbach (Aron y Aron, 2001), el cual mostró un valor de 0,873 para el instrumento total y confiabilidad mayor a o,70 en las dos escalas que lo integran (ver tabla 1), lo que indica que es un instrumento confiable. Esta versión fue posteriormente adaptada a formato electrónico utilizando la aplicación Google Form, para que fuese distribuido y respondido por vía remota.

La versión final del instrumento incluyó 21 preguntas tipo Likert de cinco puntos, donde 1 corresponde a "Totalmente en desacuerdo" y 5 a "Totalmente de acuerdo" (anexo 1). La regla de medición para interpretar la escala se planteó así: a mayor puntaje, mayor grado de acuerdo con las afirmaciones de la dimensión correspondiente, y a menor puntaje, el grado de acuerdo se consideró negativo o en desacuerdo (Blanco y Alvarado, 2005).

Las preguntas están estructuradas en relación con los dos componentes de la percepción del profesorado antes mencionados, para los cuales se considera una escala de análisis que apunta a diversos aspectos del entorno laboral de los docentes: la escala interna se planteó en relación con el modo como el profesor se ve a sí mismo, en lo personal y en lo profesional; la escala externa dio cuenta de la percepción del docente acerca de la valoración de su labor por distintos actores del sistema educativo: el Estado, la sociedad, sus estudiantes, los apoderados y el propio establecimiento donde se desempeña. La distribución de preguntas del instrumento, de acuerdo con las escalas consideradas, se detalla en la Tabla 1, donde también se incluye el valor del alfa de Cronbach por cada escala.

\section{Tabla 1. Características de las escalas de la encuesta con alfa de Cronbach}

\begin{tabular}{|c|c|c|c|}
\hline Escala & Preguntas & Alfa escala & Alfa encuesta \\
\hline Interna & $1-11$ & 0,764 & \multirow{2}{*}{0,873} \\
\cline { 1 - 3 } Externa & $12-21$ & 0,844 & \\
\hline
\end{tabular}

Fuente: elaboración propia.

\section{Procedimiento}

La recolección de datos se realizó en dos etapas. En la primera, se utilizó muestreo no probabilístico por cuotas (Hernández, Fernández y Baptista, 2006) y para ello se seleccionaron aleatoriamente 50 colegios por región, tomados de la base de datos del Ministerio de Educación, de tal forma que todas las regiones estuvieran representadas en la muestra. Se envió una carta a cada colegio seleccionado invitando a los docentes a participar del estudio e informando del objetivo del mismo y de sus características metodológicas, en apego a lo dispuesto por el Comité de Ética Institucional de la Universidad de Santiago de Chile.

En la segunda etapa, se utilizó muestreo no probabilístico con voluntarios (Giroux y Tremblay, 2004). A los docentes interesados que recibieron la invitación e información desde la dirección del establecimiento y que expresaron su interés por participar vía correo electrónico al equipo investigador 
se les envió el enlace a la encuesta para responder en línea dando el consentimiento informado al momento de enviar sus respuestas. El criterio de inclusión fue que los profesores en el último año efectivamente impartieron clases a grupos. En la encuesta se incluyeron items para indagar sexo, y región de procedencia, con el fin de caracterizar mejor la muestra.

\section{Participantes}

La muestra quedó constituida finalmente por 224 docentes de establecimientos educacionales de nivel básico y medio de todas las regiones del país, de todas las asignaturas y todas las dependencias administrativas. En la Tabla 2 se expone la distribución de la muestra en las variables sexo y región de procedencia, donde resalta un mayor porcentaje de mujeres de la región metropolitana.

Tabla 2. Frecuencia en la distribución de la muestra, por sexo y zona de procedencia

\begin{tabular}{|l|c|c|c|}
\hline & Metropolitana & Regiones & Total \\
\hline Mujer & 75 & 59 & 134 \\
\hline Hombre & 50 & 40 & 90 \\
\hline Total & 125 & 99 & 224 \\
\hline
\end{tabular}

Fuente: elaboración propia.

Respecto a la distribución, el mayor porcentaje estuvo concentrado en la región metropolitana, con un $56 \%$. En relación con el tipo de administración del establecimiento, la mayor cantidad de participantes, con un $43 \%$, corresponde a profesores de colegios subvencionados.

\section{Análisis y resultados}

Se estableció un baremo para la interpretación de la media, tal como proponen Pertuz, Rojas, Navarro y Quintero (2016) que se muestra en la tabla 3, en la que se incluyen los límites inferior cerrado [ $L i$ y el límite superior abierto $L s>$ para cada categoría o clase.
Tabla 3. Baremo para la interpretación de la media

\begin{tabular}{|c|c|c|}
\hline \multicolumn{2}{|c|}{ Intervalo } & \multirow{2}{*}{$\begin{array}{c}\text { Categorias (nivel de } \\
\text { percepción) }\end{array}$} \\
\hline $\begin{array}{c}\text { Limite } \\
\text { inferior [Li }\end{array}$ & $\begin{array}{c}\text { Limite } \\
\text { superior Ls> }\end{array}$ & $\begin{array}{c}\text { Percepción desfavorable y } \\
\text { negativa }\end{array}$ \\
\hline 1,81 & 2,71 & Percepción neutra \\
\hline 2,71 & 3,61 & $\begin{array}{c}\text { Percepción favorable y } \\
\text { positiva }\end{array}$ \\
\hline 3,61 & 4,52 & \\
\hline
\end{tabular}

Fuente: Elaboración propia.

En la Tabla 4 se presentan los resultados de las subescalas, que integran las escalas interna y externa, de acuerdo con el nivel de análisis expuesto anteriormente.

Los resultados de la subescala Personal reflejan que el $94 \%$ de los profesores consideran que aportan a la educación en su comunidad escolar ( $\left.\mathrm{P}_{1}\right)$, si consideramos las valoraciones 4 y 5 de acuerdo y total acuerdo en conjunto. No obstante, solo un 31\% está totalmente de acuerdo en que participan activamente en la toma de decisiones en educación ( $\left.\mathrm{P}_{2}\right)$, lo que, sumado al $24 \%$ que dice estar de acuerdo, sugiere una percepción favorable y positiva para esta preguntay, por ende, para toda la subescala.

Para la subescala Profesional, el resultado de la pregunta 3 respecto al sueldo recibido por los docentes muestra una alta inconformidad, lo que coincide con el resultado del estudio de Cabezas y Claro (2011). Es de interés destacar el caso de la pregunta 10, donde un $66 \%$ de los profesores está en desacuerdo con que se evalúe el aprendizaje de sus alumnos a través de pruebas estandarizadas, dada la gran cantidad de evaluaciones a las que son sometidos los estudiantes en Chile durante su escolaridad. ${ }^{2}$

Destacable es también el resultado que indica que solo el $14 \%$ de los encuestados recomenda-

2 El detalle de las diversas evaluaciones estandarizadas, nacionales e internacionales, aplicadas en Chile a nivel escolar, se puede consultar en la página de la Agencia de Educación de Chile (http://www.agenciaeducacion.cl). 
Tabla 4. Subescalas y escalas, en frecuencia y porcentaje

\begin{tabular}{|c|c|c|c|c|c|c|c|c|c|c|c|c|c|}
\hline \multirow[t]{3}{*}{ Subescala } & \multirow[t]{3}{*}{ Ítem } & \multicolumn{10}{|c|}{ Escala Likert por indicador } & \multirow[t]{3}{*}{$\mathrm{X}$} & \multirow[t]{3}{*}{ SD } \\
\hline & & \multicolumn{2}{|c|}{1} & \multicolumn{2}{|c|}{2} & \multicolumn{2}{|c|}{3} & \multicolumn{2}{|c|}{4} & \multicolumn{2}{|c|}{5} & & \\
\hline & & $\mathrm{F}$ & $\%$ & $\mathrm{~F}$ & $\%$ & $F$ & $\%$ & $F$ & $\%$ & $\mathrm{~F}$ & $\%$ & & \\
\hline \multirow[t]{2}{*}{ Personal } & 1 & $\mathrm{O}$ & $\mathrm{O}$ & 3 & 1 & 12 & 5 & 73 & 33 & 136 & 61 & 4,03 & 0,827 \\
\hline & 2 & 23 & 10 & 27 & 12 & 51 & 23 & 53 & 24 & 70 & 31 & & \\
\hline \multirow[t]{9}{*}{ Profesional } & 3 & 79 & 35 & 52 & 23 & 43 & 19 & 40 & 18 & 10 & 4 & 3,08 & 0,694 \\
\hline & 4 & 43 & 19 & 39 & 17 & 58 & 26 & 52 & 23 & 32 & 14 & & \\
\hline & 5 & 68 & 30 & 32 & 14 & 49 & 22 & 49 & 22 & 26 & 10 & & \\
\hline & 6 & 33 & 15 & 14 & 6 & 44 & 20 & 39 & 17 & 94 & 42 & & \\
\hline & 7 & 15 & 7 & 34 & 15 & 60 & 27 & 76 & 34 & 39 & 17 & & \\
\hline & 8 & 10 & 4 & 14 & 6 & 57 & 26 & 72 & 32 & 71 & 32 & & \\
\hline & 9 & 7 & 3 & 26 & 12 & 79 & 35 & 84 & 37 & 28 & 12 & & \\
\hline & 10 & 77 & 34 & 71 & 32 & 52 & 23 & 23 & 10 & 1 & $\mathrm{O}$ & & \\
\hline & 11 & 17 & 8 & 29 & 13 & 71 & 32 & 80 & 36 & 27 & 12 & & \\
\hline \multirow{2}{*}{ Estudiantes } & 12 & 5 & 2 & 22 & 10 & 34 & 15 & 83 & 37 & 80 & 36 & 4,18 & 7,95 \\
\hline & 13 & 1 & 0 & 3 & 1 & 16 & 7 & 84 & 38 & 120 & 54 & & \\
\hline \multirow[t]{4}{*}{ Establecimiento } & 14 & 16 & 7 & 22 & 10 & 47 & 21 & 69 & 31 & 70 & 31 & 3,47 & 1,0 \\
\hline & 15 & 6 & 3 & 14 & 6 & 46 & 29 & 64 & 21 & 94 & 42 & & \\
\hline & 16 & 18 & 8 & 24 & 11 & 48 & 21 & 66 & 29 & 68 & 30 & & \\
\hline & 17 & 69 & 31 & 50 & 22 & 39 & 17 & 43 & 19 & 23 & 10 & & \\
\hline \multirow[t]{2}{*}{ Sociedad } & 18 & 3 & 1 & 12 & 5 & 54 & 24 & 81 & 36 & 74 & 33 & 3,06 & 0,773 \\
\hline & 19 & 63 & 28 & 83 & 37 & 52 & 23 & 25 & 11 & 1 & $\mathrm{O}$ & & \\
\hline \multirow[t]{2}{*}{ Estado } & 20 & 90 & 40 & 78 & 35 & 38 & 17 & 17 & 8 & 1 & 0 & 1,84 & 0,867 \\
\hline & 21 & 113 & 50 & 64 & 29 & 36 & 16 & 10 & 4 & 1 & 0 & & \\
\hline Escala interna & & & & & & & & & & & & 3,25 & 0,639 \\
\hline Escala externa & & & & & & & & & & & & 3,20 & 0,684 \\
\hline Escala total & & & & & & & & & & & & 3,23 & 0,595 \\
\hline
\end{tabular}

Fuente: elaboración propia.

ría a sus estudiantes elegir carreras de pedagogía $(\mathrm{P} 4)$, porcentaje que aumenta a $37 \%$, si se agrupa la frecuencia de los valores 4 y 5 de la escala de Likert interpretados como acuerdo con la pregunta. Este dato es comparativamente más bajo que el obtenido respecto a la preferencia que declaran tener por la profesión, que los haría escogerla de nuevo si pudieran, con 59\% (P6), y la percepción positiva que tienen respecto a la formación disciplinar, de $63 \%$, y pedagógica, de 51\%, recibidas (P7 y P8).
Por otra parte, en la subescala Estudiantes se encontró que la percepción de los profesores de cómo los valoran sus estudiantes da 91\% de acuerdo en relación con la confianza que depositan en ellos como formadores (P13), siendo alto también (73\%) el número de profesores que se siente conforme con el trato que recibe de sus estudiantes. En esta subescala se encuentra la media más alta, con una valor de 4,18 (SD = 0,795), lo que sugiere, de acuerdo con el baremo establecido previamente, que la percepción es 
favorable y positiva, con dispersión intermedia. Sin embargo, en la subescala Establecimiento, se encontró que la media disminuye de manera importante, lo que se explica porque solo el $62 \%$ de los profesores está de acuerdo con el trato recibido por las autoridades del establecimiento, aunque $71 \%$ está de acuerdo en que su establecimiento confía en cada uno y en su desempeño (P15).

En el caso de la subescala Sociedad, se presenta una dualidad en la valoración como formadores, desde el punto de vista social, ya que solo un $12 \%$ de los profesores considera que la sociedad confía en su calidad como docentes (P19); no obstante, dentro de su comunidad educativa, que se puede considerar "cercana", la percepción respecto a la confianza que los apoderados, padres y madres depositan en ellos como formadores es alta (P18), con un $69 \%$ de acuerdo, al agrupar la frecuencia de los valores 4 y 5 de la escala Likert.

Finalmente, en la subescala Estado se encontró la media más baja de todo el instrumento, con un valor de 1,84 (SD = 0,867), lo que sugiere una percepción desfavorable y negativa según el baremo establecido. Este resultado se puede entender, al observar los altos porcentajes de desacuerdo que expresan los docentes: pues $75 \%$, al agrupar la frecuencia de los valores 1 y 2 de la escala Likert, considera que las autoridades no confían en la labor docente desempeñada (P2O) y $79 \%$ piensa que el Estado no respeta el rol del docente en la formación de la ciudadanía (P21). En ambos casos, el porcentaje de acuerdo (valores 4 y 5 de la escala Likert) no supera el 10\%, un resultado clave para comprender los factores que influyen en la baja valoración social del profesorado.

El resultado global de la percepción de los docentes respecto a su rol y valoración social, referida tanto a aspectos internos como externos, mostró una media de 3,23 (SD = 0,595), que se corresponde, de acuerdo con el baremo establecido previamente, con una percepción neutra, en términos cuantitati- vos. Lo anterior, después de observar el comportamiento de las subescalas y escalas, sugiere que los profesores tienen una percepción positiva y favorable en algunos aspectos de su actividad profesional (Personal, Estudiantes y Establecimiento) y una percepción desfavorable y negativa en relación con el Estado. La Figura 2 expone en términos generales el comportamiento de las escalas, donde resalta cómo los indicadores asociados a las diferentes subescalas tienen comportamientos favorables y desfavorables, que se compensan entre sí hasta provocar que la media total tome un valor que sugiere una percepción neutra.

Figura 2. Comportamiento de subescalas y escalas del estudio

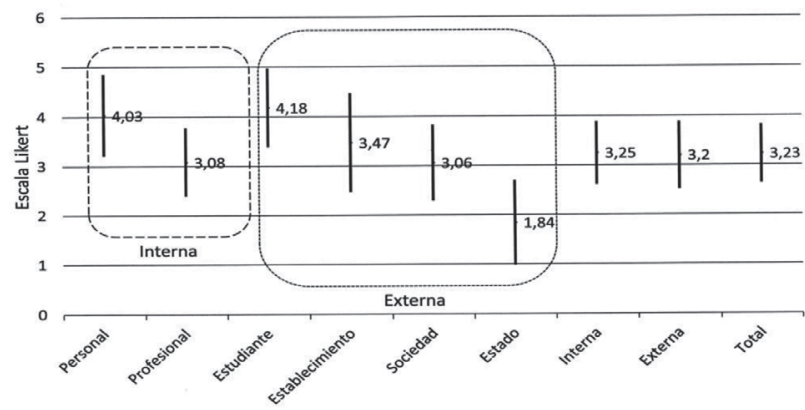

Fuente: elaboración propia.

En la tabla 5 se presenta el resumen de los valores estadísticos descriptivos de las dos escalas, que permiten definir la percepción de los docentes.

Tabla 5. Estadisticos descriptivos de las escalas externa e interna

\begin{tabular}{|c|c|c|c|c|c|}
\hline & 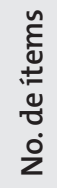 & 怘 & 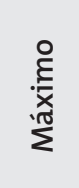 & $\frac{\pi}{\pi}$ & 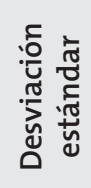 \\
\hline $\begin{array}{c}\text { Escala } \\
\text { interna }\end{array}$ & 11 & 1,64 & 4,55 & 3,25 & 0,639 \\
\hline $\begin{array}{c}\text { Escala } \\
\text { externa }\end{array}$ & 10 & 1 & 4,6 & 3,20 & 0,684 \\
\hline Total & 21 & 1,81 & 4,52 & 3,23 & 0,595 \\
\hline
\end{tabular}

Fuente: elaboración propia. 
Finalmente, para indagar en la asociación entre escalas se utilizó la correlación de Spearman (Aron y Aron, 2001), la cual resultó positiva moderada entre las escalas interna y externa $\left(r_{p}=0,630, p<\right.$ $0,01)$. Este resultado sugiere que en ambas escalas se reproduce el fenómeno neutro de compensación entre aspectos favorables y desfavorables de la percepción. Como se observa en la Figura 2, en la Escala interna, la subescala Personal-que tiene una media que se puede interpretar como favorable- se compensa con la media de la subescala Profesional, que se interpreta como percepción neutra. En el caso de la Escala externa, la subescala Estudiantes -que se interpreta como una percepción favorable de acuerdo con el valor de la media-se compensa, entre otras subescalas, con el promedio de Estado, que presenta el valor más bajo de todo el instrumento y, por tanto, arroja una percepción desfavorable.

\section{Conclusiones}

A partir del análisis realizado, se ha identificado que existen diferencias en la percepción del profesorado respecto a su rol y valoración social, en función del contexto respecto al cual se consultó, determinado por los factores considerados en la escala interna y externa. La lectura cuantitativa de estas diferencias indica que los resultados globales son neutros, lo cual es reflejo de complementariedad entre las subescalas definidas (Novoa, 2009). No obstante, en una interpretación cualitativa, es posible sugerir una tensión entre los diversos factores que influyen en la construcción global de la identidad profesional docente.

Por una parte, existe una valoración interna positiva que está dada por la percepción que tienen los profesores del reconocimiento y valoración recibida de sus estudiantes y apoderados, en su entorno cercano y sobre su formación profesional. Este resultado es coherente, de manera recíproca, con el encontrado en estudios similares (Arancibia, 1994), donde la percepción de la opinión pública respecto al profesor resultó favorable.
Si bien se identificó que ser profesor da satisfacción personal en relación con su responsabilidad social, esto ocurre en desmedro de la baja compensación económica recibida. Es decir, la valoración positiva que perciben los profesores de su rol como formadores se contrapone a la baja valoración percibida por el Estado y la sociedad en general. En este sentido, la llamada Identidad Profesional Docente (Ávalos et al., 2010) puede considerarse favorable en los profesores encuestados, mirando desde su percepción sobre la profesión y labor desarrollada.

En coherencia con el estudio de Cabezas y Claro (2011), los resultados reflejan que, si bien los profesores volverían a estudiar pedagogía, no lo recomiendan para sus estudiantes y aún menos para sus hijos. Esta situación sugiere que hay un factor emocional o afectivo de la persona hacia la profesión, que podría ser de tipo motivacional y que también contribuye en la explicación de por qué los factores internos contrarrestan los externos, relacionados con el reconocimiento social o la retribución económica.

En términos generales, y dado que la percepción negativa de la valoración social proviene mayormente del ámbito del Estado, queda de manifiesto la posibilidad de que las representaciones sociales sobre la profesión docente contribuyan a su desvalorización social, más que la propia percepción de los profesores, quienes valoran positivamente su rol, desempeño, formación y contribución a la sociedad. Por ende, se requiere mayor reconocimiento, retribución e incentivo por parte del Estado para que en la valoración social del profesor se alcance el estatus que este merece. Para ello se requiere de políticas públicas que contribuyan a mejorar las condiciones laborales de los docentes a corto, mediano y largo plazo, dada la repercusión directa que esto puede tener en la valoración social del profesorado para complementar la percepción favorable tanto interna como cercana al docente. En esta línea, la nueva política de carrera docente que actualmente se discute en Chile, de ser consensuada con el profesorado, podría 
aportar a mejorar la valoración social del profesorado en su dimensión externa.

Se sugiere, como prospectiva de este estudio, explorar cada subescala por separado para justificar las diferencias detectadas en el detalle que podrían estar relacionadas con aspectos motivacionales presentes en la representación social de la pedagogía en Chile, entre otros no considerados en este estudio, tales como la satisfacción por el propio desempeño y la formación recibida, que resultan de interés para considerar en la elaboración de políticas públicas que favorezcan la atracción de nuevos talentos hacia la profesión.

Desde el punto de vista de las limitaciones del estudio, se considera de interés poder replicar el estudio en el futuro, para una muestra más grande de docentes en ejercicio, y que pueda complementarse con un enfoque cualitativo que indague por los motivos de la percepción aquí identificada. Dado que el estudio fue realizado en línea, no hubo instancia para profundizar en las respuestas, por lo que sería interesante entrevistar a algunos profesores encuestados para ello.

Finalmente, los resultados descritos confirman la tendencia de estudios anteriores que han explorado la percepción docente (Ávalos, 2001; Núñez, 2003; Donoso, 2008b; Cabezas, 2011), lo cual conlleva reflexionar sobre el impacto que han tenido, o no, las sucesivas innovaciones estatales realizadas en los últimos años en la valoración social del profesorado chileno. Al respecto, los resultados encontrados en este estudio plantean la necesidad de investigar acerca de las causas del porqué la percepción de la valoración social de los docentes desde el Estado continúa siendo negativa.

\section{Referencias}

Abell, S. (2000). International perspectives on science teacher education. En S. K. Abell (ed.), Science Teacher Education: An international perspective (pp. 3-6). Norwell, MA: Kluwer Academic.

Arancibia, V. (1994). La educación en Chile: percepciones de la opinión pública y de expertos. Estudios Públicos, $54,125-150$.

Arévalo, A., Cornejo, R., Reyes, L. y Sánchez, R. (2010). Ser docente y subjetividad histórica en el Chile actual: Discursos, prácticas y resistencias. Revista Polis, 9(27), 269-292.

Aron A., y Aron, E. (2001). Estadistica para psicología (2 ed.). Buenos Aires: Pearson.

Ávalos, B. (2001). Chile: Mejoramiento de la formación inicial de profesores: conjunción de políticas nacionales e iniciativas institucionales. Perspectivas - Revista Trimestral de Educación Comparada - Unesco, 49-58. [Los formadores de jóvenes en América Latina: Desafios, experiencias y propuestas] Recuperado de http://www. ibe.unesco.org/fileadmin/user_upload/archive/curriculum/LatinAmericanNetworkPdf/maldorep.pdf

Ávalos, B. (2002). Profesores para Chile: historia de un proyecto. Santiago: Ministerio de Educación.

Ávalos, B., Cavada, P., Pardo, M. y Sotomayor, C. (2010). La profesión docente: temas y discusiones en la literatura internacional. Estudios pedagógicos, 36(1), 235-263. Valdivia. 
Ávalos, B. y Sevilla, A. (2010). La construcción de la identidad profesional en los primeros años de docencia: evidencia desde la investigación. Centro de Investigación Avanzada en Educación, Universidad de Chile.

Blanco, N. y Alvarado, M. E. (2005). Escala de actitud hacia el proceso de investigación científico social. Revista de Ciencias Sociales, 11(3).

Cabezas, V. y Claro, F. (2011). Valoración social del profesor en Chile: ¿Cómo atraer a alumnos talentosos a estudiar pedagogía. Temas de la Agenda Pública, 6(42) (enero). Pontificia Universidad Católica de Chile, Facultad de Educación.

Campodónico, R. H. (2007). El movimiento estudiantil secundario chileno de mayo-junio de 2006: la actuación del poder gubernativo desde una visión macropolítica de la educación. Educere, 11(37), 271-281.

Córdoba, C. (2014). Elección de escuela en sectores pobres de Chile. Simposio Políticas Educacionales y Gestión de Sistemas Escolares - pens/edu-1. Recuperado de http://www.psicoperspectivas.cl/index.php/psicoperspectivas/article/viewFile/301/314

Donoso, S. (2008a). Políticas de perfeccionamiento de los docentes en Chile 1990-2005: silencios y proyecciones. En C. Bellei, D. Contreras y J. P. Valenzuela (eds.), La agenda pendiente en educación (pp. 79-105). Santiago: Universidad de Chile.

Donoso, S. (2008b). El perfeccionamiento docente en Chile (1990-2007): ¿Estado versus mercado? Revista Brasileira de Educação, 13(39), 437-454.

Flores, L. y Retamal, J. (2012). Clima escolar y gestión compleja del conocimiento: desafíos para la investigación educativa y la política pública en violencia escolar. Magis. Revista Internacional de Investigación en Educación, 4(8), 319-338.

Giroux, S. y Tremblay, G. (2004). Metodología de las ciencias humanas. México: Fondo de Cultura Económica.

Hernández, R., Fernández, C.y Baptista, P. (2006). Metodología de la investigación (4 ed.). México: McGraw-Hill.

Melgarejo, L. M. V. (1994). Sobre el concepto de percepción. Alteridades, 4(8), 47-53.

Novoa, A. (2009). Para una formación de profesores construida dentro de la profesión. Revista de Educación, $350,203-218$

Núñez, l. (2003). El profesorado, su gremio y la reforma de los noventa: presiones de cambio y evolución de la cultura docente. En C. Cox (ed.), Politicas educacionales en el cambio de siglo (pp. 455-517). Santiago: Editorial Universitaria.

Ortiz, I. (2012). En torno a la validez del Sistema de Medición de la Calidad de la Educación en Chile. Estudios Pedagógicos, 38(2), 355-373. Valdivia.

Pedraja, L., Araneda, C., Rodríguez, E., y Rodríguez, J. (2012). Calidad en la formación inicial docente: evidencia empírica en las universidades chilenas. Formación Universitaria, 5(4), 15-26. 
ISSN 0123-1294 | e-ISSN 2027-5358 | Educ.Educ. Vol. 20. No. 3 | Septiembre-diciembre de 2017 | pp. 434-447.

Universidad de La Sabana | Facultad de Educación

Pertuz Peralta, V. P., Rojas Caicedo, G. Y., Navarro Rodríguez, A. y Quintero, L. T. (2016). Perfil docente y fomento de la cultura del emprendimiento: búsqueda de una relación. Educación y Educadores, 19(1), 29-45.

Robalino, M. (2005). ¿Actor o protagonista? Dilemas y responsabilidades sociales de la profesión docente. Revista Prelac, 1(1), 7-23.

Rubilar, F. (2015). Formación de profesores en Chile: Una mirada crítica ante la encrucijada histórica de los cambios educacionales. Praxis Educativa, 19(1), 12-19.

Vaillant, D. (2010). La identidad docente. La importancia del profesorado. Revista Novedades Educativas, 22(234).

Valenzuela, J. R. y Flores, M. (2011). Fundamentos de investigación educativa. eBook. Tecnológico de Monterrey. 


\section{Anexo 1. Preguntas del instrumento construido}

Escala de valoración: 1 = Totalmente en desacuerdo; 2 = En desacuerdo; 3 = Ni en acuerdo ni en desacuerdo; 4 = De acuerdo; 5 = Totalmente de acuerdo.

\begin{tabular}{|c|c|c|c|c|c|}
\hline Ítems & 1 & 2 & 3 & 4 & 5 \\
\hline $\begin{array}{l}\text { 1. Siento que en mi nivel y área de experticia estoy aportando a la mejora de la educación en mi } \\
\text { comunidad escolar. }\end{array}$ & & & & & \\
\hline $\begin{array}{l}\text { 2. Siento que puedo participar activamente en la toma de decisiones educacionales de mi comunidad } \\
\text { escolar. }\end{array}$ & & & & & \\
\hline 3. Estoy conforme con el sueldo que recibo. & & & & & \\
\hline 4. Recomendaría a mis estudiantes que estudien carreras de pedagogía. & & & & & \\
\hline 5. Recomendaría a mis hijos/as que estudien carreras de pedagogía. & & & & & \\
\hline 6. Si pudiera elegir nuevamente su profesión, ¿volvería a escoger pedagogía? & & & & & \\
\hline $\begin{array}{l}\text { 7. Estoy conforme con la formación PEDAGÓGICA (didáctica, metodológica, curricular, etc.) entregada } \\
\text { por la universidad y/o instituto de formación profesional. }\end{array}$ & & & & & \\
\hline $\begin{array}{l}\text { 8. Estoy conforme con la formación DISCIPLINAR (matemática, arte, lenguaje, biología, etc.) entregada } \\
\text { por la universidad y/o instituto de formación profesional. }\end{array}$ & & & & & \\
\hline 9. Me considero un experto/a en temas de educación. & & & & & \\
\hline $\begin{array}{l}\text { 10. Considero que las pruebas estandarizadas PSU o SIMCE evalúan realmente el aprendizaje de mis } \\
\text { estudiantes. }\end{array}$ & & & & & \\
\hline $\begin{array}{l}\text { 11. Logro abarcar durante el año escolar los contenidos declarados en las Bases Curriculares para la } \\
\text { asignatura que imparto. }\end{array}$ & & & & & \\
\hline $\begin{array}{l}\text { 12. Estoy conforme con el trato recibido por parte de los estudiantes en mi rol de docente dentro del } \\
\text { aula. }\end{array}$ & & & & & \\
\hline 13. Siento que los estudiantes confían en mí para formarlos, debido a mi calidad profesional. & & & & & \\
\hline 14. Estoy conforme con el trato recibido por las autoridades del establecimiento donde trabajo. & & & & & \\
\hline $\begin{array}{l}\text { 15. Siento que el establecimiento educacional donde trabajo confía en mí y en la calidad de mi } \\
\text { desempeño. }\end{array}$ & & & & & \\
\hline $\begin{array}{l}\text { 16. Siento que en el establecimiento educacional donde trabajo dispongo de flexibilidad para tomar } \\
\text { decisiones y proponer intervenciones educativas que promuevan aprendizajes de calidad en el } \\
\text { estudiantado. }\end{array}$ & & & & & \\
\hline $\begin{array}{l}\text { 17. Considero que la carga horaria que tengo en el establecimiento permite desempeñarme } \\
\text { adecuadamente en todas las labores que se me solicitan. }\end{array}$ & & & & & \\
\hline 18. Siento que los apoderados, padres y madres, confían en mí para formar a sus hijos. & & & & & \\
\hline 19. Siento que la sociedad chilena, en general, confía en la calidad de sus docentes. & & & & & \\
\hline $\begin{array}{l}\text { 20. Siento que el Estadoy sus autoridades confian en la labor docente desempeñada por el profesorado } \\
\text { chileno. }\end{array}$ & & & & & \\
\hline $\begin{array}{l}\text { 21. Siento que el Estadoy sus autoridades respetan el rol del docente en la formación de la ciudadanía } \\
\text { de Chile. }\end{array}$ & & & & & \\
\hline
\end{tabular}


